\title{
Integralidade do cuidado no pneumopata crônico: uma perspectiva psicológica
}

\author{
Integrality of care in chronic lung disease patients: a psychological perspective
}

\author{
Priscila Ferreira Friggi ${ }^{[a]}$, Ângela Barbieri ${ }^{[b]}$, Marisa Pereira Gonçalves ${ }^{[c]}$, Alberto Manuel Quintana ${ }^{[d]}$
}

\footnotetext{
${ }^{[a]}$ Mestranda em Psicologia pela Universidade Federal de Santa Maria, UFSM, Santa Maria, RS Brasil, e-mail:

prifriggi@hotmail.com

${ }^{[b]}$ Mestre em Psicologia pela Universidade Federal de Santa Maria, UFSM, Santa Maria, RS Brasil, e-mail:

angelacherobini@yahoo.com.br

${ }^{[c]}$ Doutora em Ciências da Saúde pela Universidade de Brasília, UNB, Professora do Departamento de Fisioterapia e Reabilitação da UFSM, Brasil, e-mail: masapg61@yahoo.com.br
}

${ }^{[\mathrm{d}]}$ Pós-Doutor em Bioética pela Universidade Complutense de Madrid, Espanha, Professor do Departamento de Psicologia e Programa de Pós-Graduação em Psicologia da UFSM, e-mail: albertomquintana@yahoo.com.br

Recebido: 18/05/2013 Received: 05/18/2013

Aprovado: $12 / 11 / 2013$ Approved: 11/12/2013

\section{Resumo}

0 vertiginoso aumento das condições crônicas constitui-se uma problemática com contornos continentais, sendo responsável por cerca de 36 milhões de mortes em todo o mundo. Entre as doenças crônicas, destaca-se as de ordem respiratória, chamadas pneumopatias, que afetam diretamente as vias aéreas superiores e inferiores. Nesse contexto, observam-se intercorrências na qualidade de vida dos indivíduos acometidos, gerando limitações físicas e emocionais, bem como grande impacto econômico e social. Diante disso, compreendendo a importância de viabilizar novas modalidades de assistência em saúde, surgiu o propósito de atuar de forma multidisciplinar no atendimento ao pneumopata crônico. Assim, esse estudo trata-se de uma pesquisa descritiva qualitativa, realizada em um hospital público do interior do Rio Grande do Sul, que objetivou verificar se o acompanhamento psicológico aliado à intervenção multiprofissional diminuiria os níveis de ansiedade e depressão observados em pneumopatas crônicos. Participaram quatro sujeitos, de ambos os sexos, com dispneia, de 30 a 70 anos, apresentando níveis de ansiedade e depressão. Para a triagem, utilizaram-se as Escala de Dispneia (Medical Research Council), Escala de Ansiedade de Beck e Escala de Depressão de Hamilton. 0 acompanhamento psicológico contemplou 10 sessões e deu-se através da Psicoterapia Breve. Posteriormente, dois participantes passaram do grau moderado de ansiedade ao leve e o restante, dos graus leve e grave, passaram ao mínimo. Quanto à depressão, três sujeitos passaram do grau leve à ausência de sintomatologia e um do grau grave ao leve. Observou-se que a intervenção psicológica favoreceu o surgimento de recursos internos para lidar com as limitações ocasionadas pelas pneumopatias crônicas.

Palavras-chave: Atenção integral. Pneumopatias. Psicologia. Ansiedade. Depressão.

\begin{abstract}
The dramatic increase of the chronic conditions constitutes a problem with continental contours, accounting for about 36 million deaths worldwide. Among the chronic diseases, we highlight those of respiratory order, called lung diseases, which affect the upper and lower airways. In this context, complications in the quality of life of the affected individuals, causing physical and emotional limitations, as well as major economic and social impact, are observed. Therefore,
\end{abstract}


understanding the importance of enabling new health care modalities, emerged the purpose of acting in a multidisciplinary way in the care of chronic lung disease. Thus, this study is a qualitative descriptive research conducted in a public hospital in a country town of Rio Grande do Sul, Brazil, that aimed to verify if the psychological accompaniment combined with multiprofessional intervention would decreased the levels of anxiety and depression observed on chronic lung disease patients. There were four participants, of both sexes, with dyspnea, aged between 30 and 70 years, presenting levels of anxiety and depression. For screening, the Dyspnea Scale (Medical Research Council), Beck's Anxiety Inventory and Hamilton's Depression Rating Scale were used. The psychological accompaniment included 10 sessions and was made through Brief Psychotherapy. Later, two participants went from moderate to mild anxiety level, and the other two from the mild and severe levels to the minimum level. As for depression, three subjects went from mild to normal level and the other one from severe to mild level. It was observed that the psychological intervention facilitated the emergence of internal resources to deal with the limitations caused by chronic lung disease.

Keywords: Integral attention. Lung diseases. Psychology. Anxiety. Depression.

\section{Introdução}

O vertiginoso aumento das condições crônicas constitui-se uma problemática com contornos continentais, sendo que o último relatório da Organização Mundial da Saúde (2012) aponta que as doenças crônicas não transmissíveis (câncer, diabetes, hipertensão, entre outras) foram responsáveis por cerca de 36 milhões de mortes em todo o mundo. Estimativas assinalam que, em 2020, 80\% das doenças nos países em desenvolvimento se originarão de problemas crônicos.

Dentre as doenças crônicas, destacam-se as de ordem respiratória (pneumopatias), que são explicadas por Fitipaldi (2009) como enfermidades que vem aumentando em prevalência, particularmente entre as crianças e os idosos, e afetam tanto as vias aéreas superiores como as inferiores. 0 autor expõe que, dentre as pneumopatias que acometem as vias inferiores, as mais comuns são as Doenças Pulmonares Obstrutivas Crônicas (DPOC), Bronquiectasia, Fibrose Cística, Asma e Tuberculose. Nesse contexto, observam-se intercorrências na qualidade de vida dos indivíduos acometidos, o que gera incapacidade e grande impacto econômico e social. As limitações físicas, emocionais e intelectuais que surgem com a doença, têm conseqüências na vida do paciente e de toda sua família (OMS, 2003).

Diante disso, compreendendo a importância de viabilizar novas modalidades de práticas de assistência em saúde, surgiu o propósito de atuar de forma multidisciplinar no atendimento ao pneumopata crônico. Essa proposta rompe com a fragmentação do sistema de saúde público através de ações mais humanizadas e baseadas na integralidade do cuidado e da atenção. Essa abordagem revela sua importância diante do fato de que essa população é mais suscetível a isolamento social e ao abandono de atividades antes desenvolvidas (Godoy et al. 2005). Isso se deve às diferentes limitações e restrições presentes nessas doenças, que podem gerar baixa qualidade de vida tanto ao usuário quanto aos seus familiares.

Nesse sentido, quando o cuidado é realizado no contexto hospitalar, na perspectiva de Merhy e Cecílio (2003), deve ser de natureza multidisciplinar e resultado da conjugação de diversos profissionais e atenção integral. Assim, configura-se necessária uma abordagem completa e holística de cada sujeito com necessidades de saúde que precise de cuidados hospitalares em algum momento de sua vida.

Partindo disso, percebe-se a relevância de contemplar esses sujeitos de uma maneira global, isto é, compreender também as implicações psicológicas geradas por suas limitações físicas e a consequente queda de produtividade e autoestima. Ressaltase, assim, a vulnerabilidade orgânica dos usuários com pneumopatias crônicas, principalmente os que apresentam o sintoma de dispneia, que diante de fatores estressores podem apresentar exacerbações graves da doença, implicando diretamente no 
aumento dos níveis de ansiedade e depressão, não raros nessa realidade.

Assim, o presente estudo trabalhou com a hipótese de que o atendimento psicológico individual, aliado à atuação interdisciplinar realizada pela Fisioterapia, Fonoaudiologia e Enfermagem, poderia reduzir os efeitos das limitações dos usuários de saúde com pneumopatias crônicas. Dessa forma, essa pesquisa objetivou verificar se o acompanhamento psicológico juntamente à intervenção multiprofissional possibilitaria a diminuição dos níveis de ansiedade e depressão observados nos sujeitos participantes.

\section{Método}

Esse trabalho constitui-se de uma pesquisa descritiva de cunho qualitativo, realizada em um ambulatório de fisioterapia e reabilitação de um hospital público do interior do Rio Grande do Sul. A pesquisa descritiva consiste em descrever características de determinadas populações ou fenômenos (Gil, 2002). A abordagem qualitativa, segundo Turato (2005), visa proporcionar o entendimento e a interpretação dos sentidos e das significações referentes a um fenômeno e não é apenas um modelo que atende a certas demandas, mas diz respeito a algo que não é visível ao olhar comum.

Inicialmente, participaram desse estudo vinte $\mathrm{e}$ seis (26) usuários de saúde de ambos os sexos, com diagnóstico de pneumopatia crônica, com dispneia, na faixa etária de 30 a 70 anos, com sintomas de ansiedade e depressão e residentes no município cujo hospital está localizado. 0 período de vínculo desses sujeitos com o ambulatório variou de 08 meses a 16 anos, até o momento desse estudo.

Para a coleta de dados gerais dos usuários participantes foi utilizada como instrumento uma ficha onde constavam nome, idade, diagnósticos pneumológicos e tempo de acompanhamento no ambulatório. Posteriormente, para a triagem e avaliação inicial do estudo, foram utilizadas a Escala de Dispneia (MRC), a Escala de Ansiedade de Beck e a Escala de Depressão de Hamilton. A escala MRC (Medical Research Council) foi aplicada pela fisioterapeuta da equipe e é composta por cinco itens, sendo que o indivíduo escolhe o item que corresponde ao quanto a dispnéia limita suas atividades cotidianas (Kovelis et al.,2008).
A Escala de Ansiedade de Beck foi construída para medir sintomas de ansiedade e foi validado no Brasil por Cunha (1999). O material constitui-se de um questionário contendo 21 itens com afirmações que são avaliadas numa escala de 4 pontos, em ordem crescente de sintoma de ansiedade.

Já a Escala de Avaliação de Depressão de Hamilton foi validada no Brasil em 1985 pela AMBAN (Ambulatório de Estudo da Ansiedade), e é amplamente utilizada para avaliar e quantificar o elenco sintomatológico apresentado por pacientes portadores de transtornos de humor. Também serve para diagnosticar quadros depressivos e possui três versões: 17, 21 e 24 itens, sendo que nesse estudo utilizou-se a primeira (17 itens).

Após a triagem, os usuários de saúde que não apresentaram dispneia, e obtiveram critérios para ausência de ansiedade e depressão, foram excluídos da próxima etapa, que consistiu em acompanhamento psicológico individual, terapia fonoaudiológica para voz e deglutição, técnicas de reabilitação pulmonar pela fisioterapia e consultas de enfermagem. Assim, chegou-se ao número de sete (07) participantes, sendo que apenas quatro (04) manifestaram o interesse de iniciar o acompanhamento psicológico. Este último aconteceu no período de outubro a dezembro de 2011, uma vez por semana, em dias e horários pré-estabelecidos com os usuários, totalizando 10 sessões com duração de 50 minutos cada, nas dependências do ambulatório. Esta intervenção se deu através da Psicoterapia Breve, que, na ótica de Santos (1997) contempla um tratamento de duração inferior a uma psicoterapia clássica, apresentando objetivos definidos e centrados em atividade, planejamento e foco. Tratando-se desse estudo, os objetivos dos atendimentos concentraram-se em potencializar maneiras de lidar com as adversidades impostas pela doença crônica, e não apenas em seus aspectos negativos e limitações. Ao encontro disso, essa técnica envolve a criação de um vínculo transitório entre o terapeuta e seu paciente, baseado na relação dialógica e na empatia (Santos, 1997; Hegenberg, 2005).

Ao término do período de acompanhamento, os participantes foram submetidos a uma reavaliação, por meio das Escalas Beck e Hamilton, a fim de observar se ocorreram mudanças nos graus de ansiedade e depressão presentes na avaliação inicial. Ocorreram, também, sessões devolutivas aos 
participantes com o intuito de esclarecer questões referentes aos resultados das testagens utilizadas inicialmente e após o término do acompanhamento.

Os dados foram obtidos através das falas dos participantes durante as sessões de acompanhamento psicológico. Eles assinaram um termo de consentimento livre e esclarecido autorizando esses registros, que foram feitos em material particular da pesquisadora. Para analisar os dados, utilizou-se a técnica da análise de discurso. Para Orlandi (2007), é através dessa técnica que se procura interpretar o real sentido do discurso do sujeito, por meio de uma observação minuciosa do pesquisador. 0 sujeito é considerado na sua história, seu contexto, diante daquilo que diz e do que não diz. A mesma autora ainda refere que essa abordagem ultrapassa o sentido das falas para que se possa construir uma análise mais coerente.

Cabe ressaltar que esse trabalho foi elaborado de acordo com as diretrizes e normas regulamentadas de pesquisa envolvendo seres humanos, atendendo à resolução no. 196, de 10 de outubro de 1996, do Conselho Nacional de Saúde do Ministério da Saúde, e foi aprovado pelo comitê de ética e pesquisa da instituição ${ }^{1}$, sob o C.A.A.E 0304.0.243.000-11.

\section{Análise dos Resultados}

Os participantes desse estudo serão aqui identificados como: Sujeito A; Sujeito B; Sujeito C e Sujeito D. Destes, A e B eram do sexo masculino e $C$ e D do sexo feminino. Destes, os sujeitos A, C e D apresentavam Bronquiectasia, sendo que o último também tinha diagnóstico de Asma Brônquica, e o sujeito B era portador de Doença Pulmonar Obstrutiva Crônica (DPOC). Os resultados dessa pesquisa demonstraram que as sintomatologias de ansiedade e depressão apresentaram importante melhora nos participantes.

Sob avaliação da Escala de Ansiedade de Beck, os sujeitos A e C apresentaram ansiedade em grau moderado na avaliação inicial, o sujeito B grau leve e o D apresentou intensidade grave. Posteriormente ao acompanhamento psicológico, os sujeitos $\mathrm{B}, \mathrm{C}$ e D passaram ao grau leve de ansiedade e o sujeito A atingiu o grau mínimo. De acordo com os resultados

\footnotetext{
1 Universidade Federal de Santa Maria - UFSM
}

observados na aplicação da Escala de Avaliação de Depressão de Hamilton, percebeu-se que os sujeitos $\mathrm{A}, \mathrm{B}$ e $\mathrm{C}$ demonstraram sintomatologia depressiva de intensidade leve e o sujeito $\mathrm{D}$, grau grave na primeira avaliação. Após o período de intervenção, observou-se que os usuários A, B e C apresentaram ausência de sintomas relativos à depressão e o $\mathrm{D}$ chegou ao grau leve.

Esses resultados evidenciaram a relevância do acompanhamento psicológico realizado, não de maneira isolada, mas concomitante a uma atenção global direcionada aos participantes dessa pesquisa através das demais atuações profissionais (Fisioterapia, Enfermagem e Fonoaudiologia). Encontram-se no trabalho de Mattos (2004) considerações sobre a integralidade do cuidado, constituindo-se na medida em que a pessoa é compreendida na sua totalidade, considerando-se os aspectos biológicos, psicológicos e socioculturais, apresentando, mesmo na dimensão individual, um caráter completo.

Isso vem ao encontro do que foi observado durante a realização dessa pesquisa, pois os usuários com doenças pulmonares crônicas requerem atenção não apenas aos sintomas físicos, inerentes às patologias que apresentam, mas também é imprescindível que se atente para todas as esferas de suas vidas e na maneira que reagem e lidam diante das limitações e restrições enfrentadas em seu cotidiano. A doença crônica traz consigo diferentes incumbências, que, geralmente, interferem no modo de viver das pessoas e dos que convivem com ela (Mattos, 2004).

Diante dessa realidade, trabalhou-se através de um constante compartilhamento de saberes entre os profissionais que atuaram nesse estudo, ou seja, pela lógica da interdisciplinaridade. Esta é caracterizada por Mendes, Lewgoy e Silveira (2008) pelo grau de integração entre as disciplinas e a intensidade de trocas entre os especialistas, onde todas as disciplinas precisam sair enriquecidas. Isso explica as diferentes atuações específicas que aconteceram concomitantemente nessa pesquisa, fomentadas por diálogos, discussões e escolhas de melhores abordagens de trabalho em prol da assistência ao usuário pneumopata crônico. Para os mesmos autores, esta lógica não é apenas uma justaposição ou ajuntamento de saberes, mas sim estabelece entre si uma interação muito mais forte.

Essa ideia corrobora com o que foi observado durante as sessões de acompanhamento psicológico 
individual, nas quais os sujeitos verbalizaram as limitações com as quais convivem e o quanto estas contribuem para a baixa qualidade de vida e, muitas vezes, afetam as relações familiares e externas. Dentre essas queixas, destacou-se a dispneia como fator importante no processo de diminuição e até mesmo extinção de algumas atividades antes desenvolvidas pelos usuários, seja em ações laborais ou de lazer.

Evidencia-se o exposto na fala "eu fico nervosa pensando que vai me dar falta de ar, quando eu tenho as crises né, aí eu fico bem ansiosa" (Sujeito C). Nesses casos, o próprio temor da dispneia implica diretamente no aumento do sintoma da ansiedade, isto é, em função desse temor, as pessoas evitam esforços físicos, aumentam seu descondicionamento e criam um círculo vicioso (D. Godoy \& R. Godoy, 2002).

Já quanto às atividades desenvolvidas anteriormente à doença, nota-se "antes eu fazia tudo dentro de casa, mas desde quando começaram os sintomas eu faço pouco, parando pra descansar toda hora" (Sujeito C). Nesse aspecto, Romano (2008) menciona que, ao descrever os sintomas, muitas vezes, o paciente os associa com determinadas circunstâncias e relata o quanto suas atividades e relações foram afetadas diante do aparecimento das queixas, tal como expõe o relato acima.

Nesse sentido, D. Godoy e R. Godoy (2002) pontuam que, diante dessa situação de limitações, os sujeitos se tornam amplamente dependentes da ajuda se seus familiares ou pessoas mais próximas, o que aumenta o sentimento de incapacidade e diminui, consideravelmente, a autoestima. Souza-Rabbo et al. (2010) contribuem descrevendo que há uma necessidade de reestruturação das atividades da vida, perante as quais o sujeito deve abster-se de algumas atividades diárias ou ao menos adequar-se na prática de outras. Isto foi demonstrado no fragmento "e até deixei de fazer salgados pra vender, o que eu fiz durante anos e adorava" (sujeito C). Retoma-se aqui Romano (2008), quando discorre sobre o papel da doença dentro de uma dinâmica familiar que, em muitos casos, se revela como um fracasso, até diante dessa, atingindo os compromissos de proteção e segurança presentes neste meio. Por essa razão, é importante haver entendimento por parte do núcleo familiar para os processos de readaptação física e emocional pelas quais o usuário com doença pulmonar crônica vai enfrentar. Assim, torna-se imprescindível que ambos visualizem e compreendam as novas condições de saúde que se apresentam, para que, dessa forma, possam conviver e lidar com o contexto de vida trazido pela doença (Ministério da Saúde, 2001).

Além disso, verificou-se também, que a doença pulmonar crônica influenciou tanto no interesse quanto no desempenho sexual, causando conflitos conjugais, conforme o relato:

Meu marido teve que se acostumar e ele entendia que tinha dias que eu não conseguia manter relação porque tinha falta de ar, isso é chato, eu imagino que pra ele não é fácil essa situação, apesar dele me dizer que entende. (sujeito D).

Essa fala, de um usuário do sexo feminino, denota a existência não apenas de uma limitação física em decorrência da pneumopatia crônica, mas a situação de constrangimento instalada diante do não exercício pleno de sua sexualidade. Segundo Bento e Gaspar (2003), essa realidade gera baixa autoestima e traz a sensação de não ser alguém capaz de seduzir ou de ser desejado, e é no contato com o outro que o corpo exerce a genitalidade e precisa ser tanto desejado quanto desejante diante dele.

Diante disso, pode-se também perceber o retorno ao ciclo vicioso dos sintomas, quando essa sensação de não ser desejado é fortemente vivenciada, o que, em algumas sessões posteriores, é revelado na fala,

Meu marido não tem mais interesse e se nega a falar sobre isso, eu nem pergunto mais pra não ficar nervosa e, quando a gente fica assim, eu percebo que a falta de ar e o aperto no peito ficam ainda mais fortes. (Sujeito D)

Nota-se o processo que se instaura, onde o cônjuge não verbaliza os motivos pelos quais não tem interesse sexual e o sujeito da pesquisa não questiona, como se houvesse um "acordo" entre ambos, sob o qual ninguém fala o que está acontecendo, criando uma situação que agrava a dispneia da paciente.

Ressalta-se o quanto essas desestabilizações, decorrentes da evolução das doenças e da forma como estas são vivenciadas, trazem como 
consequência o aumento da sintomatologia concernente à ansiedade e depressão. Nesse sentido, J. Berber, Kupen e S. Berber (2005) elucidam que a depressão prejudica não só a funcionalidade social, mas também a emocional do sujeito, que tende a isolar-se e a nutrir sentimentos de derrota e frustração.

Essas observações vêm ao encontro do fragmento:

Essa doença me deixou muito magro e tem gente que mexe comigo por causa da minha magreza. Eu sei que fica difícil conseguir alguém assim, com todos esses problemas de saúde que eu tenho e com esse aspecto. (Sujeito B).

Nesse momento, quando surgiam queixas desta ordem, era necessário possibilitar aos participantes que visualizassem outras possibilidades de produzirem e sentirem-se úteis, e não apenas enfatizar as impossibilidades que se apresentavam em períodos críticos da doença.

Percebe-se, dessa forma, que nas doenças pulmonares, são experimentados sentimentos e comportamentos diversos, resultantes de todas as alterações na capacidade física, na autoestima e na imagem corporal, principalmente nas relações com as outras pessoas e nas atividades do dia-a-dia (Martins, Franca \& Kimura, 1996). A fala citada exemplifica essa diminuição drástica da autoestima aliada ao empobrecimento da imagem corporal, como alguém que se reconhece frágil física e emocionalmente. Cabe a ressalva de que a imagem corporal de um portador de doença sofre alterações constantes, seja em decorrência de procedimentos ou de limitações físicas, conforme pontua Romano (2008). A mesma autora também refere que o processo de aceitação de um novo corpo pode gerar revolta e angústia, principalmente numa sociedade onde não são tolerados desvios de um corpo ideal e perfeito estética e funcionalmente. Uma doença sinaliza diferenças, e ser aceito é ser igual.

Diante dessas queixas trazidas pelos sujeitos participantes e suas implicações na vida diária e nas relações interpessoais, percebeu-se a relevância desse espaço de escuta que foi disponibilizado. Espaço este inserido em um ambiente hospitalar, no qual, por questões culturais e instituídas, é superestimada a queixa orgânica e há pouco lugar para a subjetividade. Destaca-se que nesses momentos surgiram verbalizações e diálogos, tanto sobre as dificuldades do cotidiano de um pneumopata crônico, quanto sobre as tentativas de lidar com elas e as maneiras encontradas por cada usuário. 0 foco principal não foi a patologia, embora seja de grande importância falar sobre ela, mas abarcou questões de todas as esferas da vida dos participantes, vislumbrando possibilidades que anteriormente não eram valorizadas pelos próprios sujeitos.

Ao término do acompanhamento, as falas eram menos arraigadas às limitações e muitas questões foram trabalhadas. Dentro do foco e do tempo previsto de atendimentos e aliado às demais atuações profissionais, foi possível atingir uma diminuição dos níveis de ansiedade e depressão verificados na testagem inicial. Um exemplo disso pode ser observado na fala "me sinto bem menos ansiosa em relação às coisas que me incomodavam antes, porque aqui eu falei, desabafei, coloquei pra fora" (Sujeito D). Outra fala semelhante, também aconteceu no último atendimento:

Conversar sobre as minhas limitações e dificuldades me fez bem, posso dizer o quanto me aliviei nesse tempo aqui, sobre assuntos que não costumo conversar com amigos, porque são coisas íntimas né. Esses sintomas, a falta de ar, a necessidade de fazer as coisas mais devagar, eu não costumo comentar isso com as pessoas de fora. (Sujeito A).

Nesse período, foi observada e reafirmada a importância de se ofertar ao usuário de saúde um lugar onde possa ser escutado, visto e onde pudesse ouvir a si mesmo. Assim, percebe-se que, em meio a uma proposta interdisciplinar, a intervenção psicológica veio para favorecer o surgimento de recursos internos para lidar com as situações adversas e é percebida a construção que cada um faz de seu quadro clínico e como isso interfere na visão que tem de si mesmo neste momento da vida. Percebe-se uma exigência de reposicionamento do paciente sobre o seu ser diante da doença instalada e de suas limitações.

Desse modo, esse estudo marca o início do programa multiprofissional que até então não havia sido implantado no referido ambulatório. Os sujeitos participantes passaram a ser assistidos integralmente e as contribuições nucleares, aliadas às práticas interdisciplinares, foram propulsoras não apenas da melhora dos sintomas concernentes à 
ansiedade e depressão, como também da aceitação das limitações impostas pela situação clínica trazida pela pneumopatia, influenciando diretamente na qualidade de vida dos usuários. Ao encontro disso, Paim (2008) afirma que a prática integral organiza e articula processos de trabalho e de tecnologias distintos, que vão além da doença e possibilitam que atuações parcelares resultem numa intervenção abrangente e completa.

\section{Considerações Finais}

Esse estudo contemplou o objetivo proposto através da observação de que a realização de acompanhamento psicológico em meio à prática multiprofissional proporcionou uma diminuição da sintomatologia de ansiedade e depressão apresentada por usuários de saúde pneumopatas crônicos. Ressaltase que o processo de conhecimento dos próprios sintomas e sensações e a oportunidade de verbalizá-los e nomeá-los trouxeram maior entendimento e compreensão diante das readaptações inerentes à doença crônica, especialmente as pneumopatias. Readaptações essas que podem ter influenciado na melhora da qualidade de vida dos participantes do estudo, possibilitando não apenas uma diminuição nos níveis de ansiedade e depressão, mas também a aceitação de que as limitações e restrições existentes podem ser menos angustiantes quando reconhecidas e não superestimadas diante de potencialidades ainda encobertas. Observou-se, através das falas registradas, que a dispneia, sintoma bastante comum em pneumopatias, pode estar relacionada ao aumento da ansiedade e da depressão, isto é, potencializa o surgimento desses sintomas em função da vulnerabilidade e debilitação que a doença crônica causa.

Nesse sentido, cabe refletir sobre a importância de proporcionar um espaço para que essas questões sejam trabalhadas e valorizadas, pois o contexto hospitalar é historicamente centrado na patologia e na visão fragmentada de corpo doente, o que não propicia intervenções como a relatada nessa pesquisa. Esse diferencial em termos de atuação profissional construiu-se visando apenas um objetivo: a atenção integral direcionada ao usuário de saúde e a valorização das singularidades. 0 foco desse estudo centrou-se em mostrar os resultados da intervenção da psicologia, especificamente; porém, não foi possível construí-lo alheio à demais atuações, tão importantes quanto a da Psicologia, no que tange aos benefícios obtidos com os usuários. No entanto, tem-se como uma limitação o número reduzido de participantes e o tempo mínimo de acompanhamento psicológico, que totalizou apenas 10 sessões, o que não possibilitou uma maior observação desses sujeitos, embora se tenham alcançado bons resultados.

Dessa forma, nota-se a relevância de expandir esse estudo, pois seus achados instigaram a desenvolver outras pesquisas, com um número superior de participantes por períodos mais prolongados de tempo. Outro aspecto a ser considerado é a questão da ausência de políticas públicas de saúde direcionadas a essa população, contemplando aspectos importantes na prevenção das exacerbações e crises remissivas das pneumopatias. 0 que acontece frequentemente e que se pôde constatar nessa pesquisa é a busca pelo atendimento na alta complexidade em tempos de crise da doença, enquanto sua manutenção poderia ser realizada em níveis primários de saúde, gerando maior autonomia e benefícios aos usuários.

\section{Referências Bibliográficas}

Amban. (1985). Ambulatório de Estudo da Ansiedade. Tradução da Escala de Ansiedade de Hamilton. São Paulo.

Bento, M; \& Gaspar, S. A. A. (2003). Adolescente cardiopata: questões ligadas à feminilidade. In: A.L.A. Ribeiro. Mulher e Coração. (pp. 19-29). São Paulo: Papirus.

Berber, J. S. S.; Kupek, E.; \& Berber, S. C. (2005). Prevalência de depressão e sua relação com a qualidade de vida em pacientes com síndrome da fibromialgia. Rev. Bras. Reumatologia, 45(2), 47-54. São Paulo.

Brasil. (2001). Ministério da Saúde. Declaração de Jacarta - Quarta Conferência Internacional de Promoção da Saúde. In: Promoção da Saúde. Brasília.

Cunha, J. A. (1999). Estudos dos pontos de corte do BDI e BAI na versão em Português. $8^{\circ}$ Congresso Nacional em Avaliação Psicológica. Pôster 78. Porto Alegre. 
Fitipaldi, R. B. (2009). Fisioterapia Respiratória no Paciente Obstrutivo Crônico. São Paulo: Manole.

Gil, A. C. (2002). Como elaborar projetos de pesquisa. $4^{\mathrm{a}}$. ed. São Paulo: Atlas.

Godoy, D. V.; \& Godoy, R. F. (2002). Redução nos níveis de ansiedade e depressão de pacientes com doença pulmonar obstrutiva crônica (DPOC) participantes de um programa de reabilitação pulmonar. Jornal Brasileiro de Pneumologia, 30(2), 121-125. São Paulo.

Godoy, D. V.; Godoy, R. F.; Junior, B. B.; Vaccari, P. F.; Michelli, M.; Teixeira, P. J. Z.; \& Palombini, B. C. (2005). 0 efeito da assistência psicológica em um programa de reabilitação pulmonar para pacientes com doença pulmonar obstrutiva crônica. Jornal Brasileiro de Pneumologia, 31(6), 499-505. São Paulo.

Hegenberg, M. (2005). Psicoterapia Breve. Clínica Psicanalítica. 2ª ed. São Paulo: Casa do Psicólogo.

Kovellis, D.; Segretti, N. O.; Probst, V. S.; Lareau, S. C.; Brunetto, A. F.; \& Pitta, F. (2008). Validação do Modified Pulmonary Functional Status and Dyspnea Questionnaire e da escala do Medical Research Council para o uso em pacientes com doença pulmonar obstrutiva crônica no Brasil. Jornal. bras. Pneumologia, 34(12), 1008-1018. São Paulo.

Martins, L. M.; Franca, A. P. D.; \& Kimura, M. (1996). Qualidade de vida de pessoas com doença crônica. Rev. Latino-Am. Enfermagem, 4(3), 670-676. Ribeirão Preto.

Mattos, A. (2004). A Integralidade na prática (ou sobre a prática da integralidade). Cad Saúde Pública, 20(5), 1411-1416. São Paulo.
Mendes, J. M. R.; Lewgoy, A. M. B.; \& Silveira, E C. (2008). Saúde e interdisciplinaridade: mundo vasto mundo. Rev Ciência \& Saúde, 1(1), 24-32. Porto Alegre.

Merhy, E. E.; \& Cecílio, L. C. O. (2003). Integralidade do cuidado como eixo da Gestão Hospitalar. Campinas: UNICAMP.

Organização Mundial de Saúde. Classificação Internacional de Funcionalidade, Incapacidade e Saúde. (2003). São Paulo: Editora da Universidade de São Paulo - EDUSP.

Orlandi, E. P. (2007). Análise de Discurso (Princípios e Procedimentos). 7a ed. São Paulo: Pontes.

Paim, J. S. (2008). Modelos de Atenção à Saúde no Brasil. In L. Giovanella; S. Escorel; L. Lobato; J. C. de Noronha; \& A. I. Carvalho. Políticas e Sistema de Saúde no Brasil. Rio de Janeiro: Fiocruz.

Romano, B. W. (2008). Manual de Psicologia Clínica para Hospitais. São Paulo: Casa do Psicólogo,

Santos, E. F. (1997). Psicoterapia Breve: abordagem sistematizada de situações de crise. $2^{\underline{a}}$ ed. São Paulo: Agora.

Souza-Rabbo, M. P.; Campos, L.; Barbosa, S. R.; Rodrigues, F. S. S.; Testa, R. F.; Dias, M. M. A.; Bosco, A. D.; Ribeiro, J. L. (2010). 0 papel de uma equipe multidisciplinar em programas de reabilitação cardiovascular. Revista Ciência em Movimento. Ano XII, n. 23. São Paulo.

Turato, E. R. (2005). Métodos qualitativos e quantitativos na área da saúde: Definições, diferenças e seus objetos de pesquisa. Rev. Saúde Pública, 39(3), 507-514. São Paulo.

WORLD Health Organization - WHO. World Health Statistics, 2012. Disponível em: <http://www. who.int/gho/publications/world_health_statistics/2012/en/index.html>. Acesso em 26 jun 2012. 